\title{
ESTUDIO DEL PENSAMIENTO TRIÁDICO EN INGENIERÍA DE SISTEMAS
}

\section{STUDY OF TRIADICAL THINKING IN SYSTEMS ENGINEERING}

\author{
MSc. Eduar Bayona Ibáñez, Andrea Natalia Bayona Moreno, \\ Juan José Cárdenas Trillos \\ Universidad Francisco de Paula Santander Ocaña, Facultad de Ingeniería, Programa de \\ Ingeniería de Sistemas, Grupo de Investigación Grucite, Semillero de Investigación \\ Siapp's. \\ Ocaña, Norte de Santander, Colombia. \\ Código postal 546552 (Vía Acolsure Sede el Algodonal), $(57+3176755473$ \\ ebayonai@ufpso.edu.co, 57+3203384640 anbayonam@ufpso.edu.co, 57+3156004103 \\ jjcardenast@ufpso.edu.co)
}

Resumen: En el presente artículo se publican los resultados de la investigación del tipo de mente de los estudiantes del programa de Ingeniería de Sistemas de la Universidad Francisco de Paula Santander, Ocaña donde se logró identificar el comportamiento del Coeficiente Tríadico (CT) con el objeto orientar a conformación de equipos de trabajo en la asignatura Introducción a la Ingeniería de Sistemas, equipos que serán evaluados una semana después de la aplicación de la prueba de Walderma de Gregori para confirmar la validez del Instrumento. Se pudo encontrar que de los 9 equipos integrados por 3 estudiantes solo uno logró buenos resultados en la consolidación de una idea de negocio.

Los resultados de la aplicación de la prueba permitió identificar el tipo de mente que predomina en los estudiantes, predomina la mente que predomina en el grupo es la de la acción también conocido como actuar, en segundo lugar el pensar y por último el sentir.

Palabras clave: Tipos de mente, Coeficiente Tríadico, investigación, Equipos

\begin{abstract}
In the present article, the results of the research of the student's mind type of the Systems Engineering program of the Universidad Francisco de Paula Santander, Ocaña are published, where the behavior of the Triadic Coefficient (TC) was identified in order to guide the conformation of work teams in the subject Introduction to Systems Engineering, teams that will be evaluated a week after the application of the Gregor's Walderma test to confirm the validity of the Instrument. It was found that of the 9 teams made up of 3 students, only one achieved good results in the consolidation of a business The results of the application of the test allowed to identify the type of mind that predominates in the students, predominates the mind that predominates in the group is the one of the action also known as acting, secondly the thinking and finally the feeling.
\end{abstract}

Keywords: Types of Mind, Triad Coefficient, Research, Equipment. 


\section{INTRODUCCIÓN}

La base conceptual de la pedagogía en las investigaciones son pocas hasta el año 2008 como lo manifiesta Mesa \& Nelson (2012) afirma que debido a que no se comprendía en profundidad la base conceptual de la pedagogía y la didáctica, hasta esa fecha siempre fueron sinónimos. La única forma de hacer investigación hasta el año 2008, más aún, La educación científica, entendida como la formación de individuos con autonomía y capacitados para investigar los fenómenos de la naturaleza y de su entorno requiere la atención de la promoción de actitudes científicas en los estudiantes como lo afirman Pérez \& Rodríguez (2011). De ahí la importancia de contar con una visión de los estudiantes que ingresan a la educación superior con el objetivo de establecer

Como lo afirma Guzmán-Luna (2014) afirma que el El Ministerio de Educación Nacional, considera como prioridad: "Elevar la calidad de la educación para que los estudiantes mejoren su nivel de logro educativo; ampliar las oportunidades educativas para reducir las desigualdades; e impulsar la investigación y el desarrollo de innovaciones educativas". De esta manera, busca ofrecer un servicio de calidad, para atender los objetivos del Plan Nacional de TIC 2008-2019, las estrategias deben estar orientas a mantener los hábitos adecuados y corregir los que son perjudiciales para el desarrollo de la cultura investigativa en el aula.

Algunos investigadores como Arias (2013) en la práctica profesional de los investigadores, en el ejercicio de la docencia, han encontrado vacíos en el proceso del aprendizaje de los estudiantes que se manifiestan en el bajo rendimiento y la deserción.

(Amaya, 2012) Las raíces de La Cognición Situada se remontan a pensadores como Vygotsky, desde donde surgen ideas que ordenan la lógica de la evolución de la consciencia y el aprendizaje humano. Este discurrir que pretende explicar la forma natural como los hombres a través de los tiempos han desarrollado procesos mentales superiores, se encuentra hoy comprimido bajo el nombre de teoría socio-cultural. Desde esta corriente se desprende esta perspectiva teórica que trata esos elementos esenciales que desde la relación hombre y contexto, posibilitan la construcción del conocimiento
En el artículo se presentan los resultados de la fase inicial de la investigación que mediante la aplicación de la de Coeficiente Triadico (CT) de Waldema de Gregori con la intención de identificar el tipo de mente que predomina en los estudiantes del programa de Ingeniería de Sistemas que inician su formación profesional, con el objetivo de contar con los criterios suficientes para conformar equipos de trabajo en la asignatura de Introducción a la Ingeniería de Sistemas con proporcionalidad adecuada entre los integrantes de los equipos y los tipos de mentes. Los aspectos relevantes de la investigación se centran en la creatividad, la lógica y la perseverancia para ser comparados con las afirmaciones de Alcaráz (2011) para identificar si existe o no relación entre los tipos de mente y la capacidad para innovar.

\section{Pensamiento Tricerebral}

El cerebro es un sistema "unitriadico" o tri-uno tres que forman uno y cada uno tan solo uno de los tres, Waldemar de Gregori afirma que anteriormente los procesos mentales se tomaban como unidades atómicas disociadas: inteligencia, voluntad, memoria, conciencia, imaginación, luego se habló especialidades de la inteligencia, como la inteligencia emocional, inteligencia financiera, inteligencias múltiples en listados lineales interminables y al azar, sin un principio ordenado, relacionador.

Anzola (2010), en la investigación titulada "Análisis de cociente triádico para procesos de admisión de estudiantes en la fundación Insutec mediante minería de datos" afirma que:

\section{Importancia del Cociente Triádico}

El cociente triádico es una herramienta de caracterización de individuos cuyo fin es analizar las variables internas del ser, las cuales al combinarse con las externas modelan un ambiente incierto y especulativo frente al comportamiento de los seres humanos en el proceso de selección y admisión de una carrera profesional. Es justo en este punto donde converge la minería de datos, un conjunto de tecnologías que permite descubrir e inferir conocimiento a partir de diferentes técnicas. (P. 3)

Danny A \& Quintero(2013), mencionan la importancia del trabajo grupal:

Son y serán pilares indispensables durante los procesos de Enseñanza/Aprendizaje. Son un 
complemento de las actividades individuales y un refuerzo del conocimiento que genera riquezas en el alumno de tipo práctico, argumentativo y analítico pues al interactuar y discutir con otros se estimulan habilidades que no se desarrollan al trabajar de manera individualizada

Para Cardona \& cardona (2012): La teoría triádica del cerebro posibilita el desarrollo de estrategias con carácter novedoso. Este es un exitoso enfoque práctico y dinámico que permite que en el aula se experimente una actividad participativa, dinámica y motivadora. La eficacia en la aplicación de este enfoque lo constituyen el desarrollo de estrategias prácticas y sencillas que enseñan al educando a pensar, sentir y actuar de manera diferente (p.77)

Waldemar de Gregori pudo hacer la exposición, en forma sistemática y didáctica, para dar a conocer y desarrollar el cerebro y sus procesos como una estructura funcional de tres bloques mínimos y sus procesos sentir, pensar y actuar o conocer, crear y hacer que conforman el Ciclo Cibernético de transformación con desempeño en cuatro (4) niveles de excelencia.

La estructura tirádica del cerebro según Waldermar de Gregori, se puede apreciar en la Figura $\mathrm{N}^{\circ} 1$

Figura $\mathrm{N}^{\circ} 1$ Tipos de mente

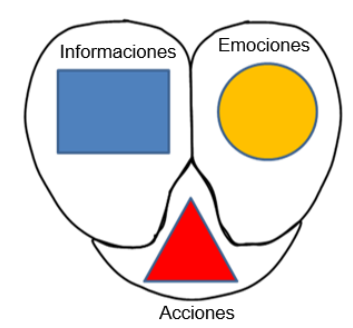

Fuente. Waldermar de Gregori

El lado racional izquierdo, representado por la figura geométrica cuadrado representa la parte del cerebro distraída por la tecnología electrónica, que no logra descifrar el caos y no consigue explicar la confusión en que vivimos, no logra tampoco abandonar su hábitos mentales y sus esquemas clasificatorios y las teorías explicativas y organizativas de otras épocas. El cerebro derecho, el proceso intuitivo, emocional, perdido en el exhibicionismo, en las supersticiones, en el milagrerismo, el lado central, operativo.

\section{El CT, Cociente Tricerebral:}

El coeficiente Intelectual o CI mide en una escala de 100 puntos. Por debajo de 100 existe una insuficiencia mental y por encima de 120 existe genialidad, según Waldermar de Gregori es una, es una escala para medir el desarrollo del potencial mental, pero casi solo del cerebro izquierdo, si se usa un test de Cociente Emocional se tiene casi la medida del cerebro derecho, desconectando el proceso intelectual, por esas razones es que el Revelador del Cociente Tricerebral permite evaluar las tres partes que conforman el cerebro que funcionan de manera integrada, sinérgica, aunque la contribución de cada una sea diferente dependiendo de su herencia genética, de la educación familiar (escolar y étnica)

La investigación busca los estudiantes de Ingeniería de Ingeniería de Sistemas de la Universidad Francisco de Paula Santander, Ocaña logren mejorar las competencias para ser gestores de su aprendizaje y de tomar parte en la generación de su propio conocimiento; además de la capacidad que se desarrolla de forma intrínseca de relacionarse adecuadamente con los miembros de su comunidad académica y establecer relaciones dialécticas y dialógicas con sus pares permitiendo la construcción argumentada de nuevo conocimiento (Mesa y Barrera, 2012).

Para Patiño (2008), manifiesta que una person con buen entrenamiento triádico:

Será alguien con predominio de un lado del cerebro sin perder la conexión y proporcionalidad con los otros dos. Será un especialista (cerebro lógico) ubicado en el contexto holístico (generalista) y que también tiene buena conexión con la realidad práctica (cerebro central). O será un generalista (predominio del cerebro (p.4)

Otro elemento de interés como lo afirma Amaya G. (2012) "desarrollar destrezas y competencias para el uso y la apropiación pedagógica de las TIC en el aula de clase" siendo vital la participación del docente.

\section{METODOLOGÍA}

El enfoque de la investigación es cuantitativa ya que se usará la herramienta CT de Waldemar de Gregori para determinar el cociente tricerebral. Siendo una escala para medir el desarrollo del 
potencial mental tipo de mente que predomina en los estudiantes que inician la educación superior, se correlacionará las variables de tipo de mente con el rendimiento académico para identificar la existencia o no de relación entre ellas.

\section{APLICACIÓN DEL TEST}

Los estudiantes del programa de Ingeniería de Sistemas se les aplicó el CT de Walderma de Gregori teniendo presente que las tres partes que integran el cerebro funcionan de forma integrada, los estudiantes deben responder con criterio las preguntas ya que es una autoevaluación. El test es subjetivo y su efectividad se comprobará en una o dos semanas de observación en su desempeño cotidiano. El test de Walderma de Gregori es una fotografía tridimensional del perfil tricerebral.

Después de que los estudiantes completen la información, se debe sumar la cantidad asignada a cada figura en su totalidad, obteniendo tres puntaje, con lo que se puede interpretar tres alternativas: 1) el CT de competencias horizontales que hace referencia a la intensidad que tiene cada uno de los tres cerebros, información importante relacionada con el desarrollo de la personalidad, 2) el CT vertical que es una radiografía de cada uno de los tres (3) cerebros en sus cuatro (4) niveles para mostrar la jerarquía de competencias verticales, indica si el desarrollo de cada cerebro está adecuado para cada ciclo de vida y 3) la CT trasversal, son dos curvas, una indica el perfil de excelencia y la otra indica el perfil de carencias

Waldemar de Gregori formula la herramienta para definir el tipo de mente que predomina en las personas, cociente triádico está conformado por un test de 27 preguntas estructuradas como se muestra en la (Fig. 2). Cada una de las preguntas pueden ser valoradas entre 13 y 5 dependiendo de la preferencia del evaluado siendo uno (1) el nivel más bajo, 3 el nivel intermedio y 5 el nivel más alto.

Fig. 2. Estructura del Test
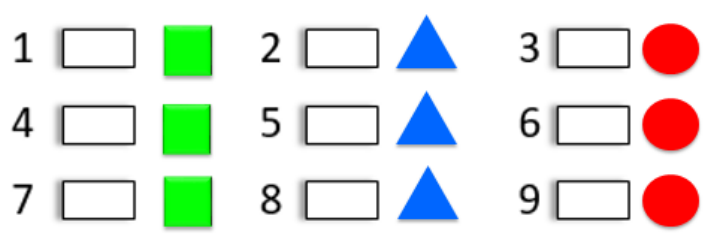

Fuente. Waldermar de Gregori
Este test fue aplicado a los estudiantes del primer semestre del programa de Ingeniería de Sistemas de la Universidad Francisco de Paula Santander Ocaña, con el cual se logró establecer el tipo de mente que predomina en cada uno, logrando así la clasificación de cada equipo de trabajo en el aula.

\section{RESULTADOS}

Resultados del test (Tabla 3) aplicado en los estudiantes de primer semestre del programa de Ingeniería de Sistemas de la Ufps, Ocaña.

Tabla 3.Tipo de mente del grupo estudiado

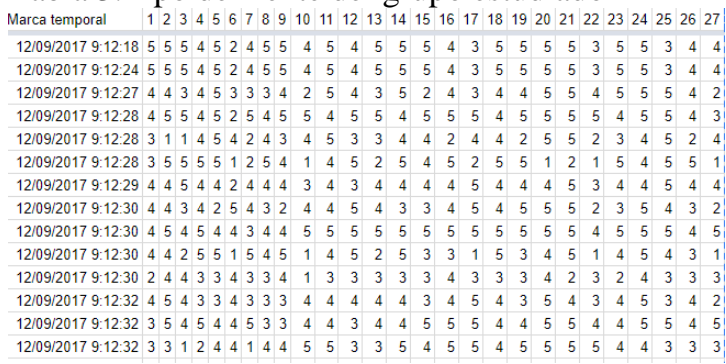
Fuente: Elaboración Propia

\section{ANÁLISIS DE LOS RESULTADOS}

Para analizar los resultados (Tabla 3), se puede observar gráficamente en el Figura 4, donde se puede apreciar existe uniformidad de la muestra, los tipos de mente son similares en los estudiantes que aplicaron el test de Walderma Degregori.

Figura 4. Análisis de resultados de la recolección de datos

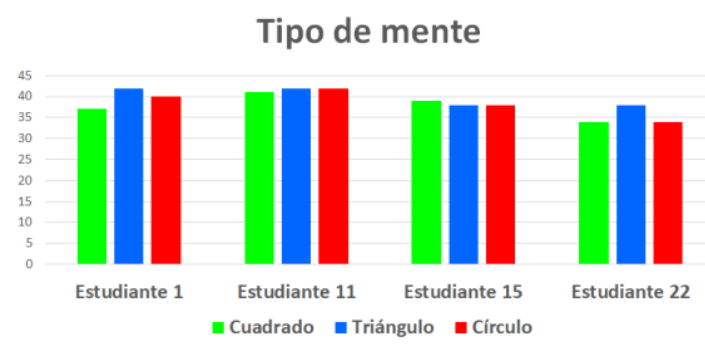

Fuente: Elaboración Propia

Los resultados muestran armonía entre las tres tipos de mente de los estudiantes de Ingeniería de Sistemas, son pocos los casos en los que se presenta bloqueo de mente por presentar los mismos valores en las tres tipos de mente, según Wladermar Degregori el estudiante que tenga los mimos resultados en los tres tipos de mente debe trabajar para entrenar su tipo de mente ya que cuando el estudiante que presente este tipo de caso al momento de ser sometido a una situación de stress se bloquea. 
Se pudo observar que en general la población que presentó la prueba cumplen con condiciones para trabajar en equipo siempre que se organicen en grupos de tres integrantes, donde cada uno de los miembros presente una tendencia bien definida en su mente, cada una las tres mentes representadas por cada una de las figuras geométricas cuadro, circulo y triangulo.

\section{CONCLUSIONES}

La aplicación de Walderma Degregori, permitió conocer el tipo de mente que predomina en los estudiantes que ingresa a la educación superior, existe un gran potencial en los estudiantes, aunque el éxito se logra con perseverancia solo un grupo logró conformar un equipo competitivo logrando los objetivos propuestos en la asignatura de Introducción al a Ingeniería de Sistemas

Los estudiantes de Ingeniería de Sistemas presentan un tipo de mente balanceado donde existe predominancia de una mente en el nivel más alto y los otros dos le siguen con valores cercanos, en el caso de las figuras geométricas del triángulo que representa a los estudiantes lideres seguido por las figuras geométricas del cuadrado para el desarrollo lógico y finalmente el circulo para los aspectos de diseño siendo ideal para conformar empresas exitosas, pero debido a que los estudiantes no son rigurosos se les dificulta mantener la StarUp en el tiempo.

Los estudiantes no son conscientes de la importancia de conformar equipos productivos en el rendimiento académico y en la conformación de empresas innovadores entorno a la tecnología como eje trasversal que les permita ser competitivos.

\section{REFERENCIAS}

Amaya, F. (2012). APROPIACIÓN Pedagógica de las Tecnologías de La Información Y Las Comunicaciones (Tic). . Revista Colombiana de tecnologías de avanzada, 59.

Anzola, J. P. (2010). Análisis de cociente triádico para procesos de admisión de estudiantes en la fundación
Insutec mediante minería de datos . 3.

Cardona, G., \& cardona, J. F. (2012). La lúdica como estrategia tricerebral para lograr aprendizajes significativos en población con dificultades de aprenidizaje. Investigaciones, 77.

Danny A, D. A., \& Quintero, S. F. (2013). Método de conformación de grupos a partir del Test RCMT. Medellin.

Patiño, M. (2008). Conozca su cerebro. Bogotá: Instituto Tecnologico Metropolitano.

Pérez, C., \& Rodriguez, S. (2011). El cerebro tríadico y su relación con la curiosidad, el trabajo en equipo y la explicación de fenómenos para el desarrollo de actitud científica. Rastros y rostros, 99.

A Arias Villa, E. Q. (2013). Relación entre la proporcionalidad cerebral triádica y el rendimiento académico de los estudiantes. Editorial IUE, 14.

Alcaráz, R. (2011). Emprendedor de Exito. México: McGrawHill.

Anzola, J. P. (2010). Análisis de cociente triádico para procesos de admisión de estudiantes en la fundación Insutec mediante minería de datos . 3.

Cardona, G., \& cardona, J. F. (2012). La lúdica como estrategia tricerebral para lograr aprendizajes significativos en población con dificultades de aprenidizaje. Investigaciones, 77.

Danny A, D. A., \& Quintero, S. F. (2013). Método de conformación de grupos a partir del Test RCMT. Medellin.

Patiño, M. (2008). Conozca su cerebro. Bogotá: Instituto Tecnologico Metropolitano. 
Pérez, C., \& Rodriguez, S. (2011). El cerebro tríadico y su relación con la curiosidad, el trabajo en equipo y la explicación de fenómenos para el desarrollo de actitud científica. Rastros y rostros, 99.

C.Cirricular. (2013). Documento maestro de ingeniería de Sistemas. Ocaña.

De Gregori, W. (2001). Bioética según la teoría cibernética social . Revista Latinoamerica de Bioética, 1-4.

Elena, C. (2008). La evaluación por competencias en la educación superior.

Enríquez Villota, M. F. (2013). Hábitos y técnicas de estudio en la Universidad Mariana. UNIMAR, 81-97.

Fabiola Cruz Núñez, A. Q. ( 2011). HÁBITOS DE ESTUDIO Y RENDIMIENTO ACADÉMICO EN. Actualidades Investigativas en Educación, 3.

Gross, E. L. (2014). Cómo parender a estudiar. Alcobendas: Libsa.

Guzmán-Luna, I. T. (2014). Propuesta De un Generador de Aplicaciones Educativas Basadas en Televisión Digital usando Arquitectura de Cómputo en la Nube . Revista Colombiana de Tecnologías de Avanzada, 33.

Mesa, L., \& Nelson, B. (2012). La Robótica Educativa como Instrumento

Hernandez, H. P., \& García, G. L. (s.f.). Enfoques, métodos y procedimeintos en la psicología del estudio .

Manuel, G. J. (1991). La formación integral: Objetivo de la universidad - Revista complutense de la educación , 323.

MEN. (2013). Linemaientos Política de Educación Superior Inclusiva. Bogotá.
MEN. (2015). Estrategias para la permanencia en eduación superior: Experiencias Significativas. Bogotá: Qualificar.

MEN. (2016). Lineamientos de política de bienestar para instituciones de educación superior. Bogotá.

MEN. (2016). Plan sectorial 2006-2010. Bogotá.

Mesa, L., \& Nelson, B. (2012). La

Robótica Educativa como

Instrumento Didáctico. Revista

Colombiana de Tecnologías de

Avanzada, 60.

Pozar, F. F. (2014). Inventario Hábitos de Estudio. Madrid: TEA Ediciones .

Torres, M., Tolosa, I., Urrea, M. d., \& Monsalve, A. M. (2009). Hábitos de estudio vs fracaso académico. Universidad de Costa Rica, 15-24.

Villegas, C. A., \& Muñoz, F. (2009). Hábitos de estudio de los alumnos en el área de Química Orgánica y su impacto en el rendimiento académico. Biotencia. 